Baltic Astronomy, vol. 9, 451-462, 2000.

\title{
DETERMINATION OF THE UNCERTAINTIES IN FREQUENCY, AMPLITUDE AND PHASE FROM MONTE CARLO SIMULATIONS
}

\author{
J. E. S. Costa and S. O. Kepler
}

Instituto de Física da Universidade Federal do Rio Grande do Sul, 91501970 Porto Alegre, RS, Brazil

Received December 8, 1999.

\begin{abstract}
Pulsation frequencies of a pulsating star appear as peaks in the frequency spectra of the star. The uncertainties of the detected frequencies may be expressed in terms of error limits based on the spectral resolution. Usually, amplitude $A$ and phase $\phi$ of a given pulsation mode, as well as their respective standard deviations, $\sigma_{A}$ and $\sigma_{\phi}$, are calculated from linear sinusoidal fit. In this work we discuss the use of Monte Carlo simulations in the determination of $\sigma_{\nu}, \sigma_{A}$ and $\sigma_{\phi}$. We show that the values of $\sigma_{A}$ and $\sigma_{\phi}$ derived from LSINF underestimate the actual values, which can endanger the correct interpretation of the statistical significance of data and results.
\end{abstract}

Key words: methods: data analysis, Monte Carlo simulations stars: pulsating stars

\section{INTRODUCTION}

The temporal changes of a pulsation $g$-mode (with symmetry axis close to the rotation axis of the star) are described by a sinusoidal function with frequency $\nu$ (or period $P=\nu^{-1}$ ), amplitude $A$ and phase $\phi$ :

$$
y(t)=A \cdot \sin (2 \pi \nu \cdot t+\phi) .
$$

In the frequency spectrum, a possible pulsation frequency appears as a peak whose height is the amplitude of the pulsation mode. Fourier transform, as well as the linear sinusoidal fit (LSINF) provide estimates for the phases: in the case of the Fourier transform, the 
phases are given by the phasors (or arguments) in the complex numbers space.

Independently of the way how $\nu, A$ and $\phi$ are determined, it is important to know the uncertainty in the estimates. The uncertainty of a measurement may be defined as an indicator of how much this measurement can differ from its actual value in terms of probability and may be specified in different ways. The two more common forms to indicate an uncertainty in the result are the standard deviation $\sigma$, and the error limit, $L$. The interpretation of the standard deviation, or error limit (as well as other forms of uncertainties) is based on the concept of confidence interval.

Suppose that $X$ is the measured value of a physical magnitude. If the true value $X_{\text {true }}$ of this magnitude has a probability $P$ of being in the interval:

$$
(X-\delta)<X_{\text {true }}<(X+\delta)
$$

where $\delta \geq 0$, then we say that the interval $[X-\delta, X+\delta]$ is a $P$ confidence interval for the magnitude $X_{\text {true }}$, and that the assertion (1) has a $P$ confidence level.

Table 1. Confidence level for some uncertainties.

\begin{tabular}{cccc}
\hline Uncertainty & Symbol & Confidence interval & Confidence \\
\hline Standard deviation & $\sigma$ & $(y-\sigma)<y_{\text {true }}<(y+\sigma)$ & $68.3 \%$ \\
$\#$ & $2 \sigma$ & $(y-2 \sigma)<y_{\text {true }}<(y+2 \sigma)$ & $95.4 \%$ \\
$\#$ & $3 \sigma$ & $(y-3 \sigma)<y_{\text {true }}<(y+3 \sigma)$ & $99.7 \%$ \\
Error limit & $L$ & $(y-L)<y_{\text {true }}<(y+L)$ & $\simeq 100 \%$ \\
\hline
\end{tabular}

By definition, $\sigma$ is the standard deviation of the error distribution of the measurements of a magnitude. The confidence levels for $\sigma, 2 \sigma$ and $3 \sigma$ for a normal (Gaussian) distribution are indicated in Table 1. Also by definition, the error limit, $L$, is the value with a confidence level close to $100 \%$. For this reason, it is usual to use $3 \sigma$ as an error limit ( $99.7 \%$ confidence level). Sometimes, the term "error" is used as synonym of "uncertainty", but in this work we define error, $\epsilon$, as the difference between an estimated value and the actual value: $\epsilon=X-X_{\text {true }}$. 
It is usual in asteroseismology of pulsating stars to calculate $\sigma_{A}$ and $\sigma_{\phi}$ (standard deviations of the amplitude and phase, respectively) through algorithms that perform a linear fit of sinusoidal curves by least squares (LSINF). This kind of algorithm does not allow the determination of $\sigma_{\nu}$, the standard deviation of the frequency. The uncertainty in frequency is usually expressed in terms of error limit: $L=\delta \nu / 2$, where $\delta \nu$ is the spectral resolution. Additionally, many authors (Winget et al. 1985, Winget et al. 1991) has pointed out that calculated values of $\sigma_{\phi}$ by LSINF appear to underestimate the actual values.

In this work we discuss the use of Monte Carlo simulations to estimate $\sigma_{\nu}, \sigma_{A}$ and $\sigma_{\phi}$. The algorithm used for this purpose is called "MCS" and is described in Section 2. In Section 3 we use a statistical test to demonstrate that the values of $\sigma_{\phi}$ and $\sigma_{A}$ derived from LSINF underestimate their actual values. In Section 4 we use MCS to show the efficiency of the sinusoidals subtraction technique to improve the measurements of $\nu, A$ and $\phi$. Finally, in Section 5 we present a few comments about the possible use of MCS in programming of observing campaigns.

\section{MONTE CARLO SIMULATIONS}

Monte Carlo simulations is a very general technique that can be used to determine the actual law of measurement errors (see Press et al. (1986) for more general explanations about Monte Carlo techniques). We developed an algorithm called "MCS" that applies Monte Carlo simulations to calculate $\sigma_{\nu}, \sigma_{A}$ and $\sigma_{\phi}$.

Main hypothesis. Monte Carlo simulations technique is based on two main hypothesis: (i) the real data set may be modeled in terms of its main parameters; ( $i i)$ the error limits of all parameter measurements are known (or may be determined interactively) and the true parameter values are within confidence intervals defined by the error limits and centered on the measured values (as shown in Table 1).

General procedure. General procedure is as follows: in each simulation, (1) the parameter values are randomly chosen within their respective error limits and (2) a synthetic (artificial) data set is made. (3) The synthetic data set is then subjected to estimator (the measurement technique) to estimate the parameter values. (4) 
The estimated parameter values are compared with the actual values (that one has used in the synthetic data): the difference between an estimated value of a given parameter and its real value is the error. The errors are computed and (5) this same process is repeated many times with new simulations until a given condition be satisfied, stopping the simulations. (6) The obtained error distributions are analysed. In general, they are normal (Gaussian) distributions with mean values close to zero, a consequence of the hypothesis ( $i i)$. Also as a consequence of hypotheses $(i)$ and $(i i)$ the standard deviation of the error distributions must be close to the standard deviation of the parameter measured values. Of course, the validity of these two consequencies depends on the validity of the hypotheses ( $i)$ and (ii).

Parameters and estimators. In the case of light curves of pulsating stars the parameters are the frequencies of the pulsation modes and their respective amplitudes and phases: $\left\{\nu_{i}, A_{i}, \phi_{i}\right\}$. The frequencies $\nu_{i}$ are usually determined from the frequency spectrum of the light curve and the amplitudes and phases may be calculated from LSINF: $A_{i} \leftarrow \operatorname{LSINF}\left(\nu_{i}\right)$ and $\phi_{i} \leftarrow \operatorname{LSINF}\left(\nu_{i}\right)$.

The synthetic light curve. In each simulation a synthetic light curve, $\left\{t_{k}, y_{k}^{\star}\right\}$, with $k=1, \ldots, N$, is made using:

$$
y_{k}^{\star}=\sum_{i=1}^{M} A_{i}^{\star} \cdot \sin \left(2 \pi \nu_{i}^{\star} \cdot t_{k}+\phi_{i}^{\star}\right)+\eta_{k}^{\star}
$$

where $\left\{t_{k}\right\}$ are the timings of the real light curve; $\nu_{i}^{\star}, A_{i}^{\star}$ and $\phi_{i}^{\star}$ are randomly chosen within their respective error limits; and $\eta_{k}$ simulates the noise (the symbol $\star$ indicates synthetic data). The error limits must be consistent with the final values obtained for $\sigma_{\nu_{i}}, \sigma_{A_{i}}$ and $\sigma_{\phi_{i}}$. For this reason, they are set in each simulation.

Noise. A random variability $\left\{\eta_{k}^{\star}\right\}$ is added to the synthetic data in order to simulate the noise present in real light curves. Usually, the random variability is generated from a Gaussian distribution characterized by a mean value zero and a standard deviation $\sigma_{0}$ (Fullerton 1986). The MCS algorithm assumes for $\sigma_{0}$ a value between zero and a maximum allowed limit, $\sigma_{\mathrm{MNL}}$ :

$$
\sigma_{0}=\alpha \cdot \sigma_{\mathrm{MNL}},
$$

where $\alpha$ is a number between 0 and $1(0 \leq \alpha \leq 1)$, called "noise factor", and 


$$
\sigma_{\mathrm{MNL}}=\left(\frac{\sum_{k=1}^{N} y_{k}^{2}}{N-1}\right)^{1 / 2},
$$

where $\sigma_{\mathrm{MNL}}$ is the standard deviation of the maximum noise level that is obtained when all signal present in the light curve is "canceled out" (for instance, through the random rearranging of the points in the light curve).

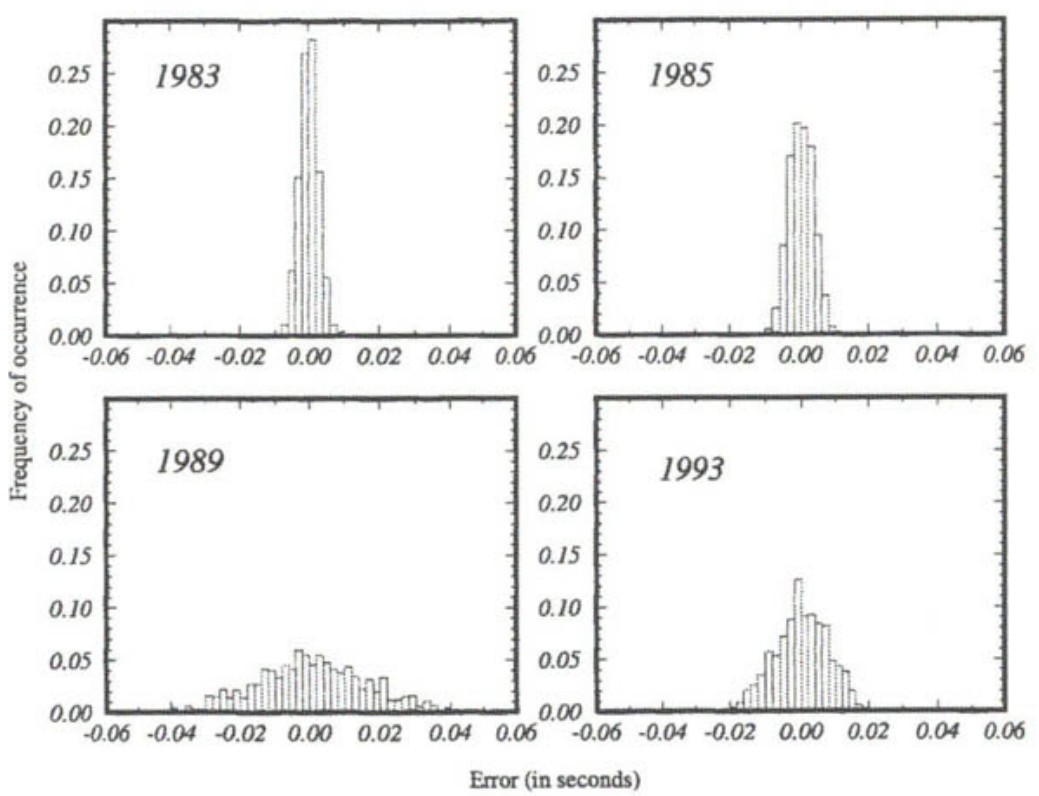

Fig. 1. Error distributions obtained from Monte Carlo simulations for estimates of the period of 516 s of PG1159-035 (number of simulations: 1000).

Errors. The next step is to obtain the frequency values from the frequency spectrum of the synthetic light curve, $\left\{\hat{\nu}_{i}^{\star}\right\}$, and then calculate $\left\{\hat{A}_{i}^{\star}\right\}$ and $\left\{\hat{\phi}_{i}^{\star}\right\}$ (the symbol ${ }^{\wedge}$ indicates the estimates of a synthetic parameter).

In general, these values are not exactly the same used in the synthetic data and the difference between the estimated value and the actual value are the errors: $\epsilon\left(\nu_{i}^{\star}\right)=\hat{\nu}_{i}^{\star}-\nu_{i}^{\star}, \epsilon\left(A_{i}^{\star}\right)=\hat{A}_{i}^{\star}-A_{i}^{\star}$ and $\epsilon\left(\phi_{i}^{\star}\right)=\hat{\phi}_{i}^{\star}-\phi_{i}^{\star}$. The errors are computed and all the process is repeated in a new simulation. 


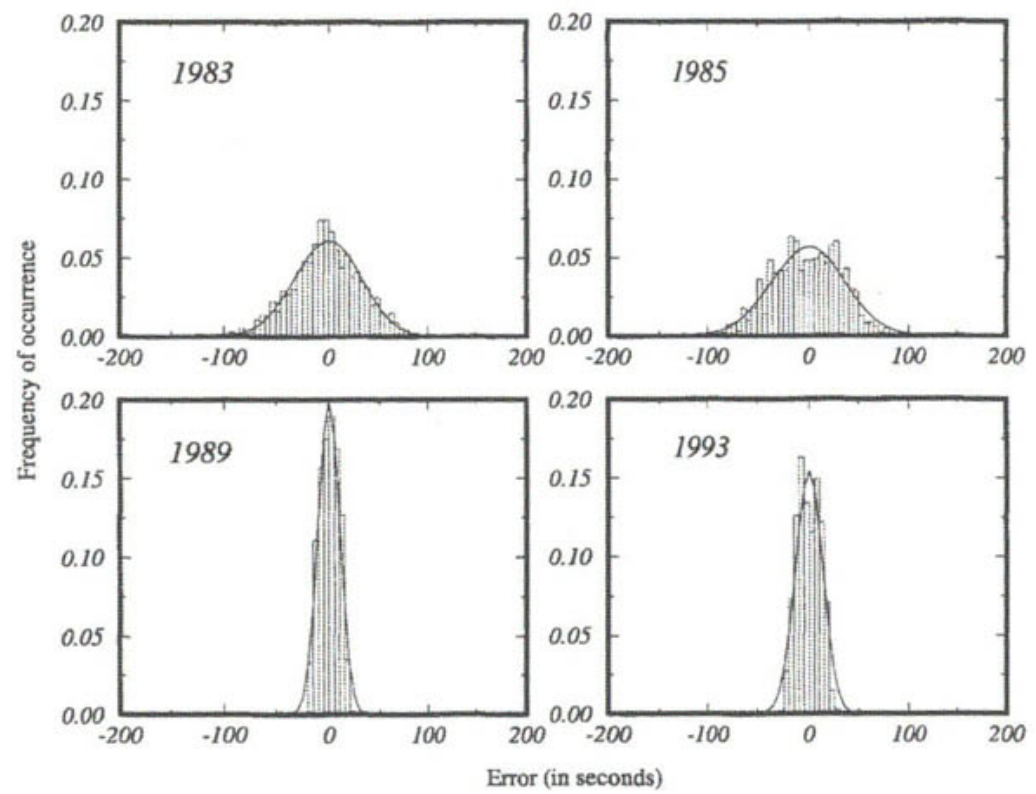

Fig. 2. Error distributions obtained from Monte Carlo simulations for estimates of the phase of the $516 \mathrm{~s}$ pulsation for four different data sets (number of simulations: 1000).

Final results. After a lot of simulations, a normal (Gaussian) error distribution is obtained for each parameter. The standard deviation of each error distribution of the parameter is assumed to be the standard deviation of estimates of this parameter for the real data. Fig. 1 shows the error distributions from 1000 Monte Carlo simulatons for estimates of the period of $516 \mathrm{~s}$ of the strongest pulsation mode in four different data sets simulating the light curves of the pre-white dwarf star PG 1159-035 obtained from WET campaigns in $1983,1985,1989$ and 1993 . Fig. 2 shows the error distributions for estimates of the phase of the same pulsation mode. Similar error distributions may be obtained for amplitude estimates.

Number of simulations. When number of simulations is increased, the values of $\sigma_{\nu_{i}}, \sigma_{A_{i}}$ and $\sigma \phi_{i}$ converge asymptotically to their final values. The MCS algorithm stops the simulations when the values of $\sigma_{\nu_{i}}, \sigma_{A_{i}}$ and $\sigma_{\phi_{i}}$ are close enough of their asymptotic limits. The asymptotic limits then may be calculated by extrapolation. 
Limitations. The identification of a peak in the frequency spectrum may be a critical point for modes with small amplitudes or when we are analysing low quality spectra (excessively "dirty" or with low resolution). In these cases the identification of a peak may be very difficult or perhaps we have to consider several peaks as possible candidates. This impose certain limitations to MCS that must be taken into account.

Relation between $\sigma_{\nu}$ and $\sigma_{P}$. From $\sigma_{\nu}$ we can calculate $\sigma_{P}$, the standard deviation of the pulsation period $(P=1 / \nu)$ and vice-versa:

$$
\begin{aligned}
& \sigma_{\nu}=\frac{\sigma_{P}}{P^{2}}=\nu^{2} \sigma_{P}, \\
& \sigma_{P}=\frac{\sigma_{\nu}}{\nu^{2}}=P^{2} \sigma_{\nu} .
\end{aligned}
$$

\section{LSINF}

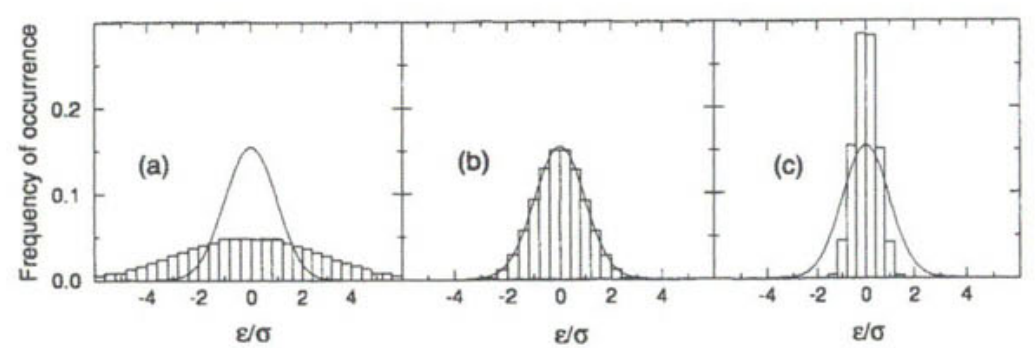

Fig. 3. Error/sigma test. Three different distributions of $\epsilon / \sigma$ values are compared with the espected curve for a Gaussian processes: in (a) the values of $\sigma$ are underestimated; in (b) the values $\sigma$ are normal; and in (c) the values of $\sigma$ are overestimated.

If the estimator calculates the $\sigma$ of a parameter its performance can be tested by computing the ratios $\epsilon / \sigma$ between the obtained error $\epsilon$ and the error $\sigma$ calculated by the estimator in each simulation. For the Gaussian process the probability distribution for $\epsilon / \sigma$ is given by:

$$
\mathcal{P}(\epsilon / \sigma)=\frac{1}{\sqrt{2 \pi}} \cdot e^{-\frac{1}{2}(\epsilon / \sigma)^{2}} .
$$


If the estimator tends to underestimate the actual values of $\sigma$, the obtained distribution of $\epsilon / \sigma$ is more "flat" than the normal distribution of Eq. (2). On the other hand, if the estimator tends to overestimates the actual values of $\sigma$, the obtained distribution is more "sharp" than the normal distribution. Fig. 3 shows the $\epsilon / \sigma$ distribution for (a) underestimated values of $\sigma$; (b) for normal values and (c) for overestimated values of $\sigma$.

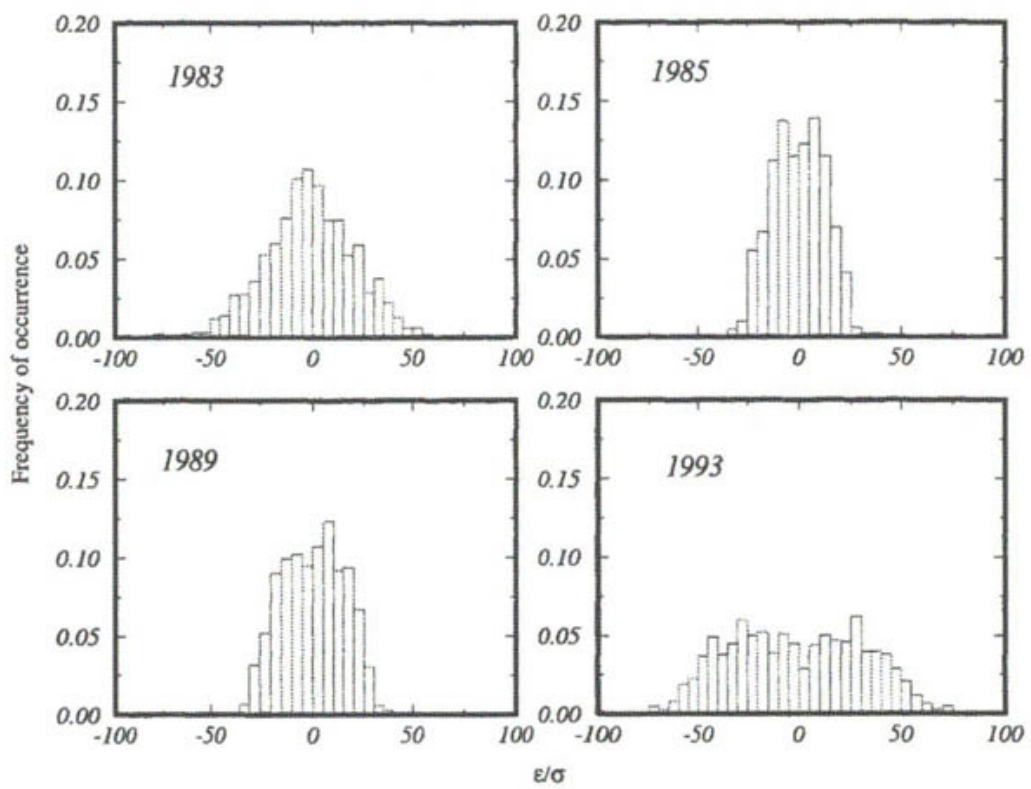

Fig. 4. Distributions of the values of $\epsilon / \sigma$ of error/sigma tests for the values of $\sigma_{\phi}$ calculated from LSINF for four different data sets (number of simulations $=1000$ ).

Both $\sigma_{A}$ and $\sigma_{\phi}$ can be calculated from LSINF. LSINF linearizes the sinusoidal function by means of a variable change, mounts a linear equation system and, after solving it, calculates $A$ and $\phi$. The values of $\sigma_{A}$ and $\sigma_{\phi}$ are derived from the covariance matrix of the equation system. This works when the light curve consists of only a sinusoid + noise, but tends to fail when there are other sinusoids, due to interference between the signals. This is the case of light curves of the multiperiodic pulsating stars. The values estimated by LSINF for $\sigma_{A}$ and $\sigma_{\phi}$ tend to underestimate the actual values (Costa et al. 
1999), which can seriously endanger the correct interpretation of the statistical significance of data and results.

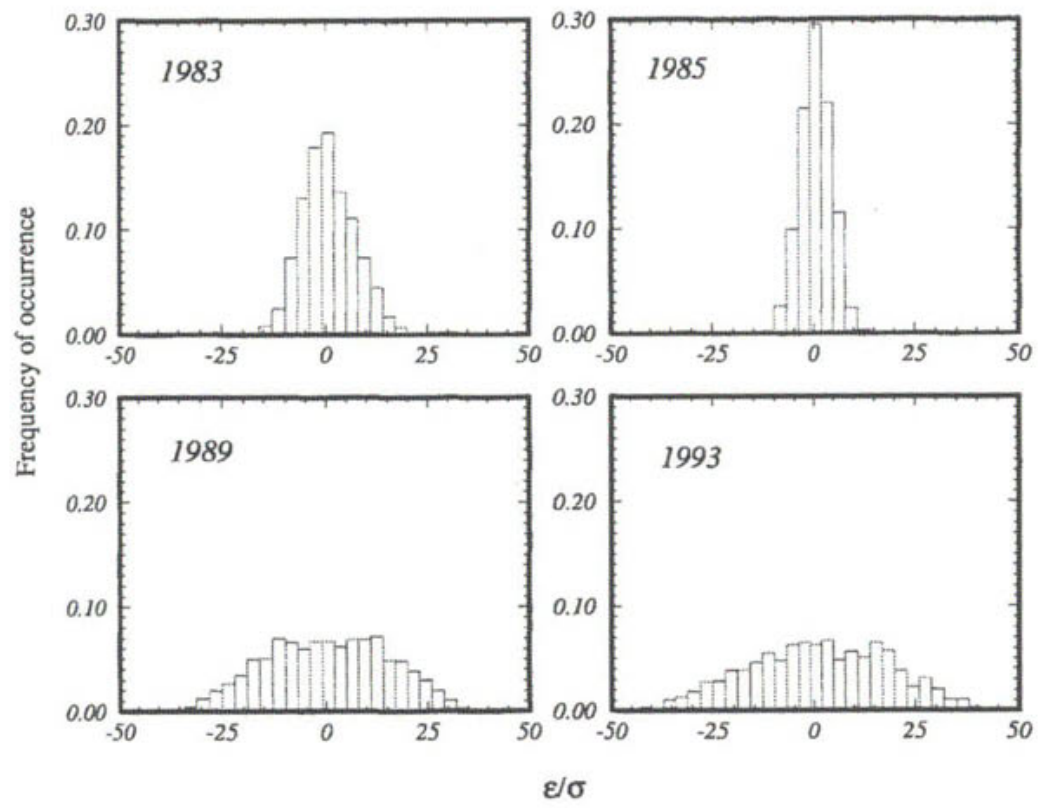

Fig. 5. Distributions of the values of $\epsilon / \sigma$ of four error/sigma tests for the values of $\sigma_{A}$ calculated from LSINF.

Fig. 4 shows the results of the error/sigma test for estimates of $\sigma_{\phi}$ from LSINF for a mode of $516 \mathrm{~s}$ of PG 1159-035 and Fig. 5 shows the results of the error/sigma test for estimates of $\sigma_{A}$ from LSINF for the same four synthetic data sets. For 1000 simulations the frequency of occurrence of $|\epsilon / \sigma|>3.5$ might be close to zero. The obtained results indicate that $\sigma_{A}$ and $\sigma_{\phi}$ from LSINF tend to underestimate strongly the true values.

\section{PREWHITENING}

A form to improve the estimates of the frequency, amplitude and phase of a sinusoid is the technique called prewhitening. It consists of subtracting from the light curve the largest possible number of other sinusoidal curves. In practice, it may be difficult to completely 
subtract a sinusoid from the light curve, but in general it is possible to reduce the amplitude of many of them, decreasing the effects of the interference and improving the estimates of $\nu, A$ ou $\phi$. The prewhitened data can be modeled and then we can apply the MCS algorithm to found the uncertainty of the new results. The efficiency of the prewhitening process is illustrated in Fig.6, that shows the error distributions in the determination of the amplitude and phase of 516 s mode of PG 1159- 035, before and after the data prewhitening.

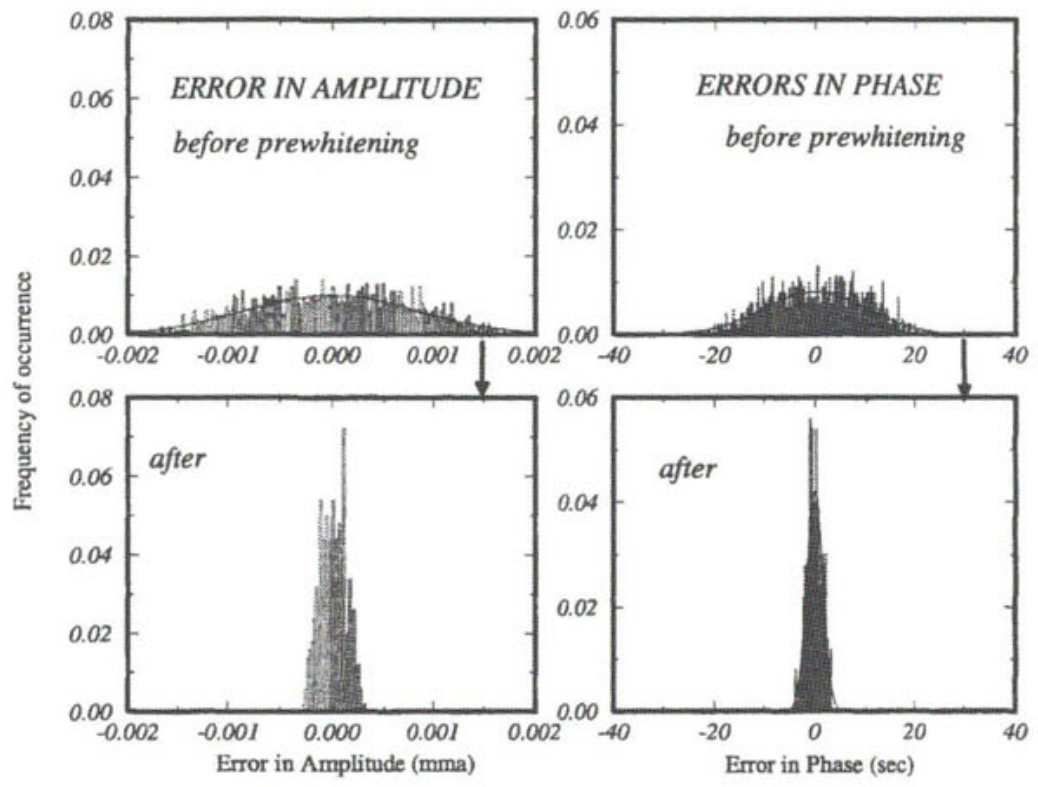

Fig. 6. Efficiency of the prewhitening. Figure shows the error distributions in calculation of amplitude (left) and phase (right) of a pulsation mode before (top) and after (bottom) prewhitening of the data.

\section{MCS \& WET}

MCS may be useful in the programming of observing campaigns. From simulations it is possible to verify if the desired accuracy may be reached or not. For high precision asteroseismological analysis the continuity of the light curves is most important. Nonetheless, simulations demonstrate that the extension of the light curve is more 
important than its continuity when we want to obtain only good estimates for the pulsation frequencies. On the other hand, the prewhitening is more efficient for more continuous data. Through MCS it is possible to find the optimum relation between the continuity and the extension of observing campaigns for a given star that minimizes the uncertainties in the determination of its pulsation period. This can be useful, for instance, in the study of changes pulsation periods of DOV stars (Costa et al. 1999).

\section{SUMMARY AND COMMENTS}

In this work we have demonstrated from the error/sigma test that the values of $\sigma_{A}$ and $\sigma_{\phi}$ calculated from linear sinusoidal fitting (LSINF) tends to strongly underestimate their actual values, which can put in danger the correct interpretation and statistical significance of the data or results. We propose to use the Monte Carlo simulations to calculate more accurate values of $\sigma_{A}$ and $\sigma_{\phi}$, and also to estimate the standard deviation of the pulsation frequencies, $\sigma_{\nu}$. Monte Carlo simulations can be employed in the data analysis of stars whose frequency spectrum already was modeled.

We also demonstrate the efficiency of the sinusoid subtraction technique (prewhitening) to improve the estimates of the frequency, amplitude and phase of the pulsation modes. Finally, we point out that, in general, the accuracy in frequency measurement depends more on the extension of the campaigns than on their continuity. This must be taken into account if we are interested to carry out direct measurements of the changes in the pulsation periods of DOVs stars.

ACKNOWLEDGMENTS. Most of the calculations were made using the Cray YMP-2E computer at the Centro Nacional de Supercomputação da UFRGS.

\section{REFERENCES}

Costa J.E. S., Kepler S. O., Winget D. E. 1999, ApJ, 522, 937

Fullerton A. W. 1986, in The Study of Variable Stars Using Small Telescopes, ed. J. R. Percy, Cambridge University Press, p. 201

Press W. H., Teukolsky S. A., Vetterling W. T., Flannery B. P. 1986, Numerical Recipes in FORTRAN, Cambridge University Press, p. 684. 
Winget D. E., Kepler S. O., Robinson E. L., Nather R. E. 1985, ApJ, 292, 606

Winget D. E., Nather R. E., Clemens J. C., Provenca J. L., Kleinman S. J., Bradley P. A., Wood M. A., Claver C. F., Frueh M. L., Grauer A. D., HineB.P., Hansen C. J., FontaineG., AchilleosN., WickramasingheD.T., Marar T. M. K., Seetha S., Ashoka B. N., O'Donoghue D., Warner B., Kurtz D. W., Buckley D. A., Brickhill J., Vauclair G., Dolez N., Chevreton M., Barstow M. A., Solheim J.-E., Kanaan A., Kepler S. O., Henry G. W., Kawaler S. D. 1991, ApJ, 378, 326 